\title{
Partitioning Gait Cycles Adaptive to Fluctuating Periods and Bad Silhouettes
}

\author{
Jianyi Liu and Nanning Zheng \\ Institute of AI \& Robotics, Xi' an Jiaotong University, P.R. China \\ jyliuaaiar.xjtu.edu.cn
}

\begin{abstract}
Period detection and cycle partitioning are always the very beginning for most gait recognition algorithms. Badly segmented silhouettes and random fluctuations in walking speed are two of the main problems for this basic but important issue. In this paper, we propose a method of cycle partitioning that is adaptive to silhouette quality and speed fluctuations. To do that, autocorrelation on sliding window is proposed to quantify the silhouette quality into "trusted zones" and "uncertain zones". Prior period estimation and observation of fluctuations are incorporated to obtain more precise cycle detection. One criterion based on the difference of Common Phase Frames (CPF) is proposed to evaluate the precision of detection. In experiment, our method was compared with the traditional autocorrelation method using sequences from the USF gait database. The results showed the improved cycle partitioning performance of the proposed method.
\end{abstract}

\section{Introduction}

As a young branch of biometrics, human gait has raised much attention in recent years. It has great potential in individual identification and video surveillance areas. Research in this field includes gait modeling [11], gait feature analysis [13], gait recognition based on model $[4,8,12,13]$ or appearance $[2,7,9,10]$, silhouette extraction and refinement $[8,10]$, and semantic gait analysis etc.

Periodicity is one of the important characteristics of gait out of other biometrics. Precise period estimation is always the very beginning of any gait recognition algorithm. The autocorrelation-based methods [1,5] are a simple but common way to compute period from the foreground sum of silhouettes. Sinusoidal signal was used in [3] to fit such foreground sum curve so that the period was ready to be read. In [4], minima of the sum signal were detected and used as the separating points of cycles. [2] investigated and quantified the impacts of 4 different walking speeds $(0.7,1.0,1.3,1.6 \mathrm{~m} / \mathrm{s})$ upon gait recognition performance, and proposed method to normalize gait features across varying period lengths. [6] estimated the stride length and cadence by assuming a linear characteristic of them under multiple walking speeds. 
The major problems of gait cycle detection lie in two aspects: badly segmented silhouettes and varying walking speeds. The first problem is mainly caused by complex background. Pre-filtering [4,5] is usually applied to de-noise the foreground sum signal prior to analysis. However, local signals with severe noise are hard to remedy through this global filtering. The second problem is varying speed, which has been paid less attention in literature up to now. Some works $[2,6]$ analyzed gait at several discrete speeds. Instead, we aim to study fluctuating walking speed, which means the speed varies randomly around an approximate constant. This reflects the real status of walking behavior.

In this article, systematical study on periodicity and cycle detection of silhouettebased gait sequences is performed: we proposed the concept as well as a prototype approach of Silhouette Quality Quantification (SQQ) using autocorrelation on sliding window. Based on the binary estimation of sequence quality along frame number, "trusted zones" and "uncertain zones" were separated. Cycle detection adaptive to fluctuating speed and silhouette quality were implemented by incorporating the prior estimation and observations in each zone. In addition, a criterion based on the mean difference of Common Phase Frames (CPF) was proposed to quantitatively evaluate the cycle partitioning precision.

Our method contributes on robust period detection even with very bad silhouette parts, and can detect the time-variation cycles of gait sequence with subtle fluctuation in walking speed. Comparing with the fixed period detection in traditional approaches, our method results in more precise cycle partitioning that is beneficial for any further gait analysis task.

\section{Fixed Period Detection}

Most of the approaches towards gait recognition detect gait cycles using foreground sum signal $S(n)=\sum_{x, y} I(x, y, n), n=0 \cdots N-1$, from all $N$ silhouette images $I(x, y, n)$. This signal is usually very noisy due to complex background so that preprocessing is required. In [4], an adaptive filter was applied prior to the calculation of gait cycles. In general, Gaussian filter [7] is a usual way to smooth the raw sum signal.

Given filtered foreground sum signal, the fixed period detection method works based on the autocorrelation function of $S(n)$, denoted as $A_{S}(n)$. Its peaks $n^{+}$can be located by judgements of $A_{S}\left(n^{+}\right)-A_{S}\left(n^{+}-1\right)>0$ and $A_{S}\left(n^{+}+1\right)-A_{S}\left(n^{+}\right) \leq 0$. The $\mathrm{x}$-coordinate of the second peak will tell the half gait period (e.g. from one double support stance to the next), denoted as prd. For simplicity, we use prd as the period value in the following.

However, the fixed period value can't precisely partition a gait sequence with fluctuating walking speed which is ordinary for normal walking. Besides, this method is also vulnerable from silhouettes with low quality. 


\section{Adaptive Period Detection}

\subsection{Silhouette Quality Quantification}

In outdoor environment, complex backgrounds make the background subtraction task difficult and produce "polluted" silhouettes, which obviously will affect the performance of appearance-based gait recognition algorithms. Therefore, methods for refining the silhouettes were proposed [8]. However, in most of the literature considering silhouette quality, the means for evaluating it still remains unsettled. We introduce a quantitative method in this paper addressing this issue.

It is easy to notice that a "clean" silhouette sequence will produce a smooth $S(n)$ curve with clear periodicity, while a "polluted" one will show a chaos $S(n)$ with vague periodicity. So $S(n)$ is a direct embodiment of silhouette quality. Because bad silhouettes often occur consecutively in real case, we study the quality of subsequence of $S(n)$ rather than single frame. Let $S i(n)$ denote the $i$-th fragment of $S(n)$. It starts from the $i$-th frame of the sequence and has a length of $W$. We can use sliding window to define such a fragment: $\operatorname{Si}(n)=S(n+i) R_{W}(n)$. Here $R_{W}(n)$ denotes the rectangle window function which has unit values for $n=0 \cdots W-1$ and zero elsewhere.

Using the same approach described in section 2, Si $(n)$ can also produce its own autocorrelation function $A_{S i}(n)$ and period value $p r d_{i}$. For walking sequence with approximately constant speed, a widely different local period $\operatorname{prd}_{i}$ apart from holistic period prd can tell a bad fragment. We use the normalized difference between these two period values as part of our fragment quality metric:

$$
Q_{x}(i)=1-\frac{\left|p r d_{i}-p r d\right|}{p r d}
$$

Another characteristic of bad fragments is their vague periodicity as mentioned above. We use the normalized difference between the heights of the first and the second peaks of $A_{S i}(n)$ to measure the periodicity here:

$$
Q_{y}(i)=1-\frac{\left|A_{S i}(0)-A_{S i}\left(p r d_{i}\right)\right|}{A_{S i}(0)}=\frac{A_{S i}\left(p r d_{i}\right)}{A_{S i}(0)}
$$

In fact, $Q_{x}$ and $Q_{y}$ are respectively derived from the horizontal and the vertical observations of the autocorrelation curve $A_{S i}(n)$. The final estimation of silhouette quality on fragment $i$ is defined as the product of them:

$$
\mathcal{Q}(i)=Q_{x}(i) \cdot Q_{y}(i)
$$




\subsection{Adaptive Cycle Partitioning}

For robust and precise cycle partitioning, we start with the fixed period value prd obtained in section 2. It serves as the prior knowledge of our period estimation and needs to be refined.

For simplicity, binary silhouette quality is used below. We binarize the $\mathcal{Q}(i)$ according to a predefined threshold th, thus dividing the whole $S(i)$ into "trusted zones" (with $\mathcal{Q}(i) \geq t h$ ) and "uncertain zones" (with $\mathcal{Q}(i)<t h$ ). $S(i)$ within the latter one is erased to decrease the impact coming from bad silhouettes:

$$
S^{\prime}(i)=\left\{\begin{array}{ll}
G(S(i)), & \mathcal{Q}(i) \geq t h \\
0, & \mathcal{Q}(i)<t h
\end{array}, \quad i=0 \cdots N-1\right.
$$

Here, Gaussian filter $G(\cdot)$ is applied upon $S(i)$ to smooth it.

In [3], a sinusoidal wave signal was used to fit the periodical foreground sum signal so that cycles of the sequence were available. Here we use a similar way and a saw wave signal $\operatorname{saw}(n), n=0 \cdots N-1$ is used. Its initial period is set to prd. We perturb its period and phase to find its optimal fitness with $S^{\prime}(i)$ by searching the max normalized cross-correlation of them. In the obtained $\operatorname{saw}^{\prime}(n)$, each peak is ready to tell a partitioning position.

Since the fragments of $S(i)$ within $\mathcal{Q}(i)<t h$ is "uncertain" and thus erased, partitioning within such zones can only rely on the prior knowledge coming from the peaks on $s a w^{\prime}(n)$. In the "trusted zones" however, the observation from $S^{\prime}(i)$ is also available. So for each prior peak position $i$ on $\operatorname{saw}^{\prime}(n)$, we search $S^{\prime}(i)$ within its $\delta$ neighbor region to find the posterior peak $n^{*}$ as depicted in (5). All these $n^{*}$ act as the partitioning points in the "trusted zones".

$$
n^{*}=\underset{n \in[i-\delta, i+\delta]}{\arg \max } S^{\prime}(n)
$$

The adaptive period detection described above can partition the cycles according to the prior rough estimation for silhouettes with bad quality, and can adjust them according to the observation from fluctuating cycle lengths for good silhouettes. So compared to the fixed period method, our method can partition the gait cycles more precisely.

\subsection{Evaluation Method}

For perfectly partitioned cycles with perfect silhouettes, all frames with the same relative offset from the start of each cycle should appear with salient similarity. Based on this idea, we built up our evaluation criterion for cycle partitioning. 
Let $n^{j}$ denote the start frame number of any cycle $j$ which occupies a phase increment of $2 \pi$. Then the frame number on phase $\varphi(0 \leq \varphi<2 \pi)$ in cycle $j$ can be computed by $n^{j}+\operatorname{round}\left(\left(n^{j+1}-n^{j}\right) \cdot \varphi / 2 \pi\right)$. The operator round $(\cdot)$ is applied here to acquire integer value. We name all such frames on the same phase of every cycle Common Phase Frames (CPF), and their frame numbers constitute set $N^{\varphi}$. The intention of defining $\mathrm{CPF}$ is to evaluate the precision of cycle partitioning according to their similarity. To do that, we firstly compute the average image of all CPFs on any phase $\varphi$ :

$$
\bar{I}^{\varphi}(x, y)=\frac{1}{\left|N^{\varphi}\right|} \sum_{n \in N^{\varphi}} I(x, y, n)
$$

Here, $\left|N^{\varphi}\right|$ means the number of elements in set $N^{\varphi}$. Similar definition as $\bar{I}^{\varphi}$ has also been used by Gait Energy Image (GEI) [9]. In our evaluation criterion, the similarity of CPFs is measured through their mean error from $\bar{I}^{\varphi}$ :

$$
e r r^{\varphi}=\frac{1}{\left|N^{\varphi}\right|} \sum_{n \in N^{\varphi}} \sum_{x, y}\left|I(x, y, n)-\bar{I}^{\varphi}(x, y)\right|
$$

In general, more precise partitioning of cycles should result in greater similarity of CPFs, that is, smaller $e r r^{\varphi}$.

\section{Experiments}

The gait sequences used in our experiments comes from the USF gait database [10]. The videos in it were captured in outdoor environment with complex background and varying illumination, so many silhouettes with low quality were produced. We randomly selected 5 sequences from the gallery set to test our method.

\subsection{Silhouette Quality}

In Fig. 1, the foreground sum signals $S(n)$ derived from two different silhouette sequences are plotted in (a) and (d). The "polluted" silhouette fragments present weak periodicity comparing to the "clean" fragments. This is the basis of our Silhouette Quality Quantification (SQQ) criterion. The quality curves $\mathcal{Q}(i)$ of (a) and (d) are showed in (b) and (e) respectively. These SQQ results present similar judgments with our subjective observations. After binarization, their respective binary silhouette quality estimations are plotted in (c) and (f). The threshold th is empirically set to 0.7 based on the characteristic of the data used in this paper. The "uncertain zones" labeled with 0 are clearly distinguishable from the "trusted zones" labeled with 1 . 
(a)

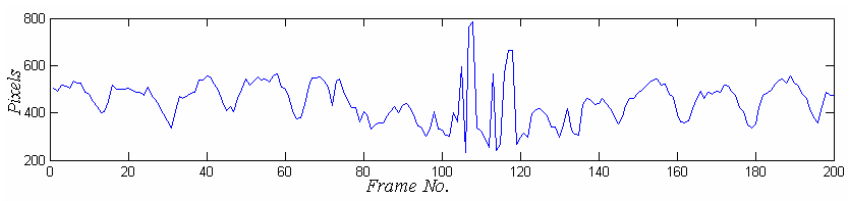

(b)

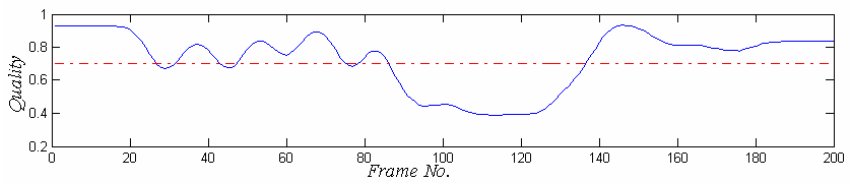

(c)

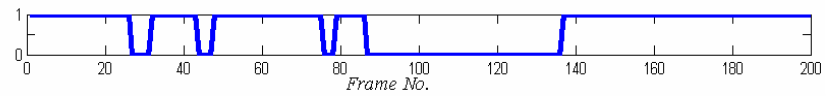

(d)

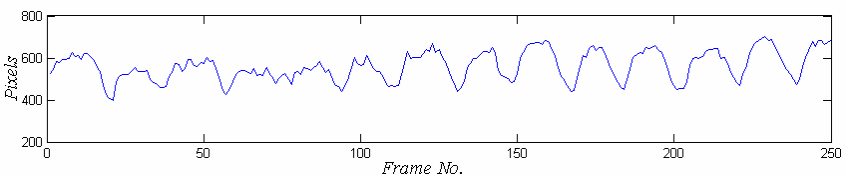

(e)

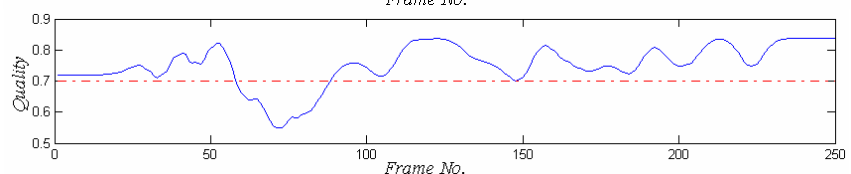

(f)

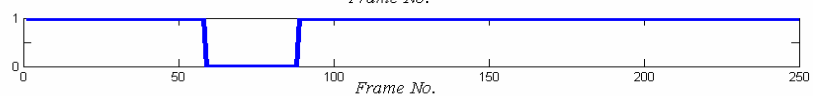

Fig. 1. Two examples of Silhouette Quality Quantification (SQQ). (a) and (d) are foreground sum signals $S(n)$ from two of our test sequences. (b) and (e) are their SQQ curves $\mathcal{Q}(i)$. (c) and (f) are their corresponding binarized results.

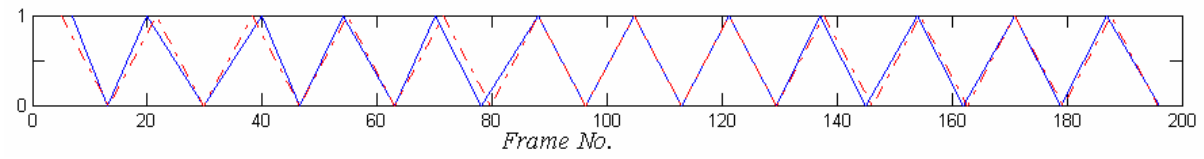

(a)

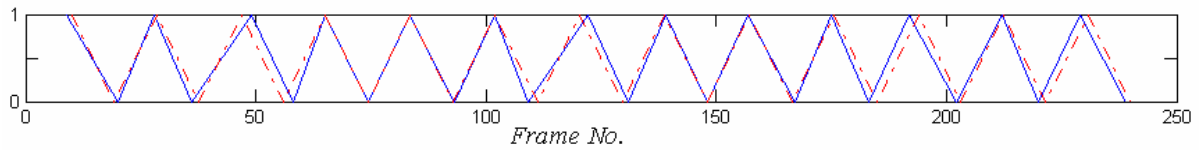

(b)

Fig. 2. Comparison of fixed cycle detection and adaptive cycle detection. (a) and (b) are from the same sequences as (a) and (d) in Fig. 1. Adaptive cycle detection is denoted by saw wave signals with solid line, while the fixed period results use the dashed line.

\subsection{Cycle Partitioning}

In Fig. 2, two examples of cycle detection results are denoted through saw waves. We use the same sequences as in section 4.1. The fixed period detections are plotted with 
dashed line, while the same sequences with adaptive cycle detection are plotted with solid line for comparison. Note that in the "uncertain zones", they overlap with each other, because only prior period value can be exploited here. However, in the "trusted zones", using neighbor region width of $\delta=2$, the prior positions of peaks have been updated by more precise observations according to (5). Same approach has also been applied to search valleys of the saw waves. The results show the characteristic of speed fluctuations in normal walking.

Table 1. The $e r r^{\varphi}$ of CPFs are used to evaluate the precision of partitioning

\begin{tabular}{|c|c|c|c|c|c|c|c|}
\hline & Phase & Seq.1 & Seq. 2 & Seq.3 & Seq. 4 & Seq. 5 & $\begin{array}{l}\text { Total } \\
\text { mean }\end{array}$ \\
\hline \multirow{5}{*}{ 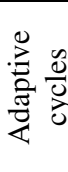 } & 0 & 6.70 & 5.42 & 7.47 & 3.19 & 5.61 & \\
\hline & $\pi / 2$ & 8.51 & 8.72 & 11.60 & 5.57 & 7.86 & \\
\hline & $\pi$ & 9.12 & 8.98 & 9.84 & 4.83 & 7.59 & \\
\hline & $3 \pi / 2$ & 7.49 & 7.79 & 8.75 & 3.45 & 4.25 & \\
\hline & mean & 7.96 & 7.73 & 9.42 & 4.26 & 6.33 & 7.14 \\
\hline \multirow{5}{*}{ 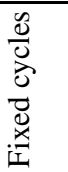 } & 0 & 7.64 & 6.48 & 8.02 & 3.16 & 5.97 & \\
\hline & $\pi / 2$ & 10.56 & 8.28 & 12.37 & 6.06 & 5.83 & \\
\hline & $\pi$ & 10.10 & 9.13 & 11.59 & 4.50 & 8.31 & \\
\hline & $3 \pi / 2$ & 8.52 & 7.70 & 8.28 & 5.12 & 4.09 & \\
\hline & mean & 9.21 & 7.90 & 10.07 & 4.71 & 6.05 & 7.59 \\
\hline
\end{tabular}

Table 1 has compared the two methods introduced in this paper. CPFs on 4 different phases $(\varphi=0, \pi / 2, \pi, 3 \pi / 2)$ from one of our test sequences are extracted and computed for evaluation of partitioning precision using the mean error criterion as (7). The proposed adaptive partitioning method outperforms the fixed period partitioning as a whole. Fig. 3 shows the CPFs produced by both methods. The results from our method (enclosed by rounded rectangles) show more consistency comparing to those from the fixed period detection.

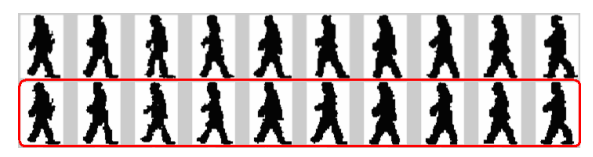

(a) $\varphi=0$

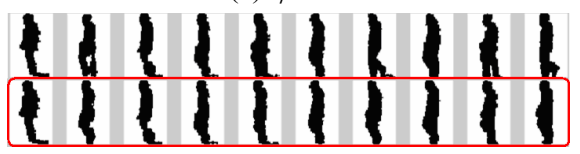

(c) $\varphi=\pi$

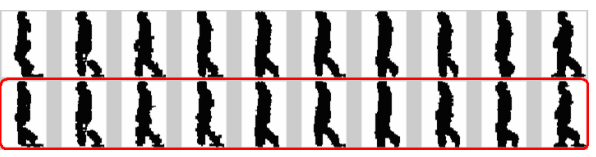

(b) $\varphi=\pi / 2$

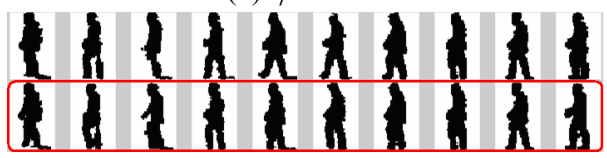

(d) $\varphi=3 \pi / 2$

Fig. 3. Eamples of CPFs on 4 phases from one of our test sequences. For each phase, the first row shows CPFs coming from the fixed period detection, while the second row enclosed by rounded rectangle corresponds to CPFs coming from the adaptive cycle detection. 


\section{Conclusion and Future Works}

In this paper, gait cycle partitioning was systematically studied, considering bad silhouette quality and fluctuating walking speed. The fixed period detection result was used as the prior estimation, which was further refined by the observation of fluctuations in the "trusted zones" to obtain more precise cycle partitioning. To do that, criteria for quantifying silhouette quality and evaluating cycle precision were proposed. The experiment results showed improved performance on partitioning precision of our method comparing with the fixed period approach.

Precision period detection is with no doubt essential to gait recognition task. Further more, the detection result of subtle fluctuations in walking speed can also provide beneficial cues for semantic and affective gait analysis. For instance, hesitating wandering will present totally different fluctuating features on walking speed compared to consistently striding. This will be our research interest next.

Acknowledgements. This work was supported by the National Science Foundation of China (Grant No. 60405004) and the National High-Tech Research and Development Plan of China (Grant No.20060101Z1059).

\section{References}

1. Boulgouris, N.V., Hatzinakos, D., Plataniotis, K.N.: Gait recognition: a challenging signal processing technology for biometric identification. IEEE Signal Processing Magazine 22(6), 78-90 (2005)

2. Tanawongsuwan, R., Bobick, A.: Modelling the Effects of Walking Speed on AppearanceBased Gait Recognition. In: IEEE Computer Society Conference on Computer Vision and Pattern Recognition, pp. 783-790 (2004)

3. Little, J., Boyd, J.: Recognizing people by their gait: The shape of motion. Videre, Int. J. Computer Vision 14(6), 83-105 (1998)

4. Sundaresan, A., Roy Chowdhury, A.K., Chellappa, R.: A hidden Markov model based framework for recognition of humans from gait sequences. In: Proc. Int. Conf. Image Processing, vol. 2, pp. 14-17 (2003)

5. Boulgouris, N.V., Plataniotis, K.N., Hatzinakos, D.: Gait recognition using dynamic time warping. In: Proc. IEEE Int. Symp. Multimedia Signal Processing, pp. 263-266 (September 2004)

6. BenAbdelkader, C., Cutler, R., Davis, L.: Stride and cadence as a biometric in automatic person identification and verification. In: 5th International Conference on Automatic Face and Gesture Recognition (2002)

7. Chai, Y., Ren, J., Zhao, R., Jia, J.: Automatic Gait Recognition using Dynamic Variance Features. In: 7th International Conference on Automatic Face and Gesture Recognition, pp. 475-480 (2006)

8. Lee, L., Dalley, G., Tieu, K.: Learning pedestrian models for silhouette refinement. In: Proc. of Ninth IEEE International Conference on Computer Vision, pp. 663-670 (2003)

9. Han, J., Bhanu, B.: Individual Recognition Using Gait Energy Image. IEEE Trans. on Pattern Analysis and Machine Intelligence 28(2), 316-322 (2006) 
10. Sarkar, S., Phillips, P.J., Liu, Z., Vega, I.R., Grother, P., Bowyer, K.W.: The Humanid Gait Challenge Problem: Data Sets, Performance, and Analysis. IEEE Trans. on Pattern Analysis and Machine Intelligence 27(2), 162-177 (2005)

11. Lu, H., Plataniotis, K.N., Venetsanopoulos, A.N.: A Layered Deformable Model for Gait Analysis. In: Proc. IEEE Int. Conf. on Automatic Face and Gesture Recognition, pp. 249256 (2006)

12. Wang, L., Tan, T., Ning, H., Hu, W.: Fusion of Static and Dynamic Body Biometrics for Gait Recognition. IEEE Trans. on Circuits and Systems for Video Technology 14(I2), 149-158 (2004)

13. Wagg, D.K., Nixon, M.S.: On Automated Model-based Extraction and Analysis of Gait. In: 6th International Conference on Automatic Face and Gesture Recognition, pp. 11-16 (2004) 